\title{
The Pattern Electroretinogram in Optic Nerve Demyelination
}

\author{
Trevor H. Kirkham and Stuart G. Coupland*
}

SUMMARY: The pattern electroretinogram (PERG) is probably generated by the ganglion cell layer of the retina whose axons constitute the conductive fibres of the optic nerves. A study was undertaken of the PERG in a group of patients with clinically definite multiple sclerosis who had suffered optic nerve demyelination to assess the value of recording the PERG as a method for electrophysiological determination of optic nerve dysfunction. Pattern visual evoked potentials (PVEP) were simultaneously recorded, each eye being stimulated separately, and four different check sizes were used. Although individual patients showed PERG abnormalities the results as a group showed no significant difference from the normal population so far as latency or amplitude of the PERG was concerned. Thus we conclude that the PERG, while an interesting measurement, does not satisfactorily reflect optic nerve dysfunction. Further, our results indicate that it is not such a useful measure as the pattern visual evoked response for detecting optic nerve disease.

RÉSUMÉ: L'électrorétinogramme évoqué par des patterns (PERG) semble avoir son origine au niveau des cellules de la couche ganglionnaire de la rétine dont les axones constituent les fibres conductrices des nerfs optiques. Chez des malades souffrant de sclérose en plaques et d'atteinte optique démyélinisante, nous avons analysé les données de l'électrorétinogramme évoqué par des patterns pour en évaluer l'utilité dans la détection des lésions du nerf optique. Bien que des électrorétinogrammes anormaux (PERG) aient été obtenus chez plusieurs malades, les différences de lantence et d'amplitude ne se sont pas révélées significatives en comparaison de celles d'un groupe témoin normal. L'électrorétinogramme évoqué par des patterns (PERG) ne réflète donc pas fidèlement la présence de démyélinisation dans les nerfs optiques; en outre, il ne se révèle pas aussi efficace que l'enregistrement des potentiels évoqués visuels obtenus par renversement de pattern pour détecter une atteinte des nerfs optiques.

Can. J. Neurol. Sci. 1983; 10:256-260

The electroretinographic responses of the cat were described to flash and pattern stimulation before and after complete optic nerve section by Maffei and Fiorentini (1981). They found evidence for two distinct electroretinograms (ERGs), that to flash stimulation (FERG), persisting indefinitely, and that to pattern stimulation (PERG) which progressively diminished in amplitude and ultimately became non-recordable after the optic nerve section. They concluded that the PERG is generated in the ganglion cell layer of the retina since its disappearance coincided with ganglion cell degeneration and development of optic atrophy after the nerve section. Although the ganglion cells may not be the actual site of PERG generation their normal function seems essential for its production. There is no evidence published for trans-synaptic degeneration of other retinal structures after optic nerve section.

We therefore decided to study the PERG in a large group of patients with well defined optic nerve dysfunction, documented both clinically and with pattern visual evoked potentials (PVEP), to compare its latency and amplitude in the diseased state with that in normal subjects.

Further we wished to determine whether recording the PERG would prove as sensitive an index of optic nerve demyelination as the pattern visual evoked potential.

\section{MATERIALS AND MeTHODS}

Patients

We examined 35 normal control subjects (15 males and 20 females) whose ages ranged from $21-35$ years. All had corrected visual acuity of $6 / 6$ and wore appropriate spectacle correction for the electrophysiological examination. All had normal Ishihara color vision, pupil reactions, Goldmann visual fields, applanation tonometric readings, ocular motility, slit lamp examination and fundi. None had suffered any ophthalmic or neurological disease.

We examined 28 patients ( 10 males and 18 females) aged between 18 - 41 years who had clinically definite multiple sclerosis (McDonald and Halliday, 1977). They had suffered unilateral or bilateral optic neuritis and had been examined in this department during at least one episode of disease and had clinical features enabling a diagnosis of optic nerve disease to be made (Kirkham and Coupland, 1981a). There were two groups of patients with clear clinical evidence of unilateral left $(\mathrm{N}=7)$ or right $(\mathrm{N}=4)$ optic nerve involvement. The third patient group $(\mathrm{N}=17)$ had bilateral involvement. These groups we called "Left ON", "Right ON" and "Bilateral ON" (ON = optic neuropathy).

From the Departments of Neurology. Neurosurgery and Ophthalmology. McGill University, and the Department of Neuro-Ophthalmology at the Montreal Neurological Hospital.

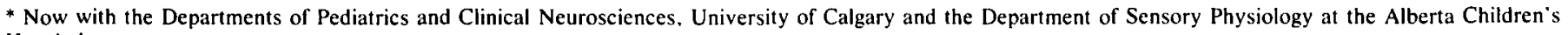
Hospital.

Received April 5. 1983. Accepted August 20, 1983.

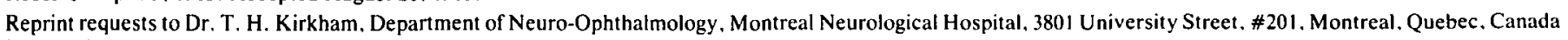
H3A 2 B4. 


\section{Stimulus Parameters}

PERGs and PVEPs were recorded to an 11 degree circular test field containing a checkerboard pattern (with pattern elements of $1.7, .7, .5, .25 \mathrm{deg} /$ side) pattern-reversed 3 times a second (reversal rate $=1.5 \mathrm{~Hz}$.). Luminances of the bright and dark checks were 200 and $20 \mathrm{~cd} / \mathrm{m}^{2}(82 \%$ contrast) determined by a Hagner S-2 Universal Spot Photometer(Optikon Corp., Waterloo, Ont.).

\section{Pattern ERG and VEP Recording}

Pattern electroretinograms (PERGs) were recorded with goldfoil hook electrodes in the lower fornix directly under the pupil and taped to the lower lid. This method is useful for elimination of electrode movement artefact from eyeblinks or eye movement (EOG). The PERG was recorded after monocular stimulation and the averaged EOG from the nonstimulated eye was digitally subtracted from the stimulated eye recording to give a purer, easily scored PERG (Coupland and Kirkham, 1981). VEPs were recorded over $0_{1}$ and $0_{2}$ with active sites referenced to linked mastoids. The raw EEG was amplified $50,000 \mathrm{x}$ by Grass P5-11J preamplifiers (Grass Instruments, Quincy, Mass.) with a .1-300 hz. bandwidth and 128 samples were signal averaged by an LS1-11 microprocessor-based TN-1710 multichannel analyzer (Tracor-Northern Instruments, Wisconsin). The resulting averaged VEP waveforms were stored on floppy disk for offline analysis.

The peak latency of the major positive (P100) component was determined for each monocular checkerboard condition. Using our normative sample mean and standard deviation a latency delay criterion exceeding $95 \%$ of normal controls was determined. Peak latency values for normal and multiple sclerosis (MS) populations were analyzed using appropriate ANOVA and nonparametric statistics. Within the MS population the relative frequency of "normal" and "abnormal" component latencies were tabulated.

\section{RESUltS}

\section{The Pattern ERG}

The mean implicit time of the $Q$ wave and peak-to-peak amplitude of the P-Q component of the PERG are presented in Table 1 and the typical waveform of the normal PERG in Fig. 1 after subtraction of eye movement artefact. Mixed ANOVA of PERG latency and amplitude measures in the unilateral and bilateral optic nerve disease groups revealed that both the within-and between-subject main effects did not reach statistical significance $(p<.05)$. Since the two unilateral optic nerve disease groups had small sample sizes another ANOVA was performed comparing only the more severely affected bilateral $O N$ group $(\mathrm{N}=17)$ with the normal subjects $(\mathrm{N}=35)$. Again there was no statistically significant difference between MS and normal group means. Also the within-subject main effect was non-significant indicating that mean latency of the $\mathrm{Q}$ component did not change systematically with decreasing check size.

In our normal subjects when we expressed the PERG interocular amplitude ratio as the smaller/larger PERG mean values of $.65-.75$ with standard deviations up to .2 were found on the four check sizes used as stimulation. Similar amplitude ratios were found in both unilateral and bilateral optic nerve disease patient groups.

\section{Pattern Electroretinograms}

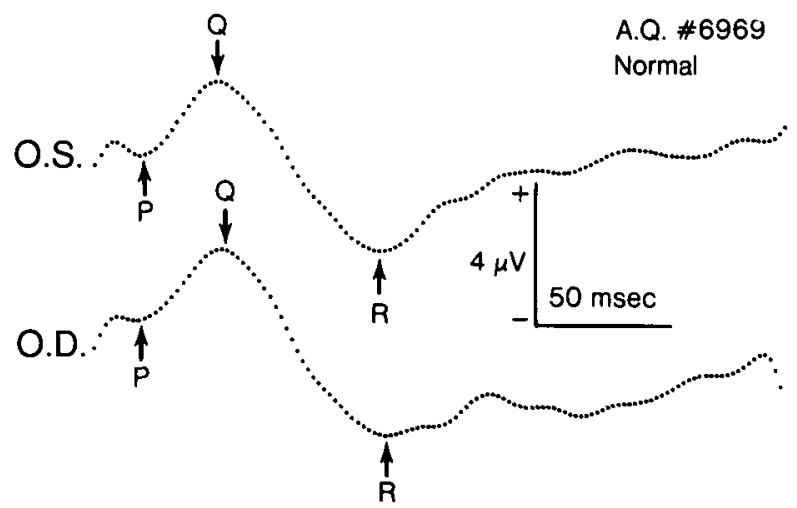

Figure I - Typicalmonocularcheckerboardpattern-reversalPERGitracings from a normal subject after digital subtraction of eye movement artefact shows excellent correspondence of waveform and peak latency of the response from the two eyes.

\section{The Pattern VEP}

The P100 component was categorized as either "normal" or "delayed" when it exceeded the statistically determined $95 \%$ criterion of the normal group values. Table 2 shows the mean peak latencies of the P100 VEP component recorded at the four check sizes. For all three MS groups the mean and standard deviation of peak latency of P100 was greater than the control group for each eye and each check size. ANOVA applied on the P100 peak latencies measured from both occipital sites indicated a statistically significant between-subject effect suggesting that PVEP peak latency is significantly delayed in the MS population as a whole. This significant finding was consistently present over both occipital leads at all four check sizes.

\section{PVEP Comparison between MS and controls}

Since the between-subject effects were found to be statistically significant, selective Scheffé post-hoc comparisons were made between treatment and control groups. These treatment group comparison results for the $\mathrm{N} 70$ and $\mathrm{P} 100$ component are presented as symbols in Table 2 indicating the significance level reached by the group contrast. At each check size the two unilateral ON groups mean latency differs significantly from the normal control group on every diseased side monocular stimulation trial but not on the normal side under monocular stimulation conditions. The slight but non-significant latency increase in the unaffected eyes from the normal control group could represent subclinical demyelination in these supposedly normal optic nerves. The bilateral OND group mean PVEP P100 component peak latencies are significantly greater than normal groups means on stimulation of either eye.

\section{Discussion}

Several observers have reported low amplitude PERG's in a variety of optic nerve diseases. Fiorentini et al (1981) used sinusoidal grating stimuli to produce PERGs reported its loss in a case of central retinal artery occlusion and a low amplitude in one patient with acute optic neuritis. Maffei (1982) observed 
Table 1: Mean peak latency of $Q$ wave amplitude of $P \cdot Q$ component of the pattern ERG as a function of check size in normal control and MS groups

\section{LEFT EYE}

LATENCY (msec) AMPLITUDE (Uv)

1.7 Degree checks

\begin{tabular}{lcc} 
Group & \multicolumn{2}{c}{ Q } \\
& Mean & sd \\
Left ON & 46.4 & 7.5 \\
Right ON & 42.0 & 9.3 \\
Bilat ON & 46.0 & 4.6 \\
NORMALS & 43.7 & 3.9
\end{tabular}

Group

Left ON

Right ON

Bilat $O N$

NORMALS

46.7

49.1

40.9

46.2

2.3
5.6
3.2
4.0

2.1

2.2

1.3

3.0

P-Q

$\begin{array}{cc}\text { Mean } & \text { sd } \\ 1.9 & 0.6 \\ 2.3 & 1.8 \\ 2.1 & 0.4 \\ 3.6 & 2.3\end{array}$

0.7 Degree checks
1.8
2.7
1.6
1.7

47.0

49.2

47.4

46.1

0.5 Degree checks

\section{Group}

Left ON

Right ON

Bilat ON

NORMALS

44.0
43.6
42.0
43.2

\section{4}

5.3

8.6

2.3

1.5
3.7
1.9
2.4

0.5
2.0
0.9
1.2

42.0

42.3

44.1

44.7

0.25 Degree checks

\section{Group}

Left ON

Right ON

Bilat ON

NORMALS

4.6
5.7
8.1
2.9

\section{RIGHT EYE}

LATENCY (msec)

AMPLITUDE (Uv)

$\mathbf{Q}$

sd
5.4
4.0
3.0
5.5

\begin{tabular}{|c|c|}
\hline \multicolumn{2}{|c|}{$\mathbf{P} \cdot \mathbf{Q}$} \\
\hline Mean & sd \\
\hline 2.1 & 1.6 \\
\hline 2.2 & 0.6 \\
\hline 2.6 & 1.1 \\
\hline 3.5 & 2.2 \\
\hline
\end{tabular}

sd

0.6

1.1

2 
contrast is significantly delayed (Table 2). We had considered that the PERG latency might be significantly delayed in our patients and provide a method to electrophysiologically dissect the visual pathway into pregeniculate and postgeniculate pathways and had hoped to more precisely determine the site of the demyelinating process in patients with MS who had PVEP delays. Clearly such dissection is not possible when the subjects are considered as a group. There were individuals who had delayed PERG components. Table 3 shows the proportion of patients with delayed PERG components and delayed PVEPs. The PVEP is obviously a much more sensitive indicator of optic nerve demyelination than the PERG.

We did not find any statistically significant difference in PERG amplitude in any patient group compared to our normal population. Even in the unilateral optic nerve disease groups the amplitude ratio of the affected/non-affected eye fell within the normal range. Thus PERG amplitude measures, like latency, are unlikely to be of value in determining the presence of optic nerve demyelination.

Sherman's (1982) finding of relatively normal PERG amplitude in optic atrophy as well as our data cast doubt on the validity of the concept that the PERG is necessarily abnormal in all patients with optic nerve disease. While the PERG may reflect ganglion cell function our data suggest that either few functioning ganglion cells are needed for it to be recordable or that it really represents the function of preganglionic retinal elements. Preliminary data by Bobak et al (1983) suggest the PERG more useful in evaluating glaucomatous (axonal type) optic nerve disease than demyelination.

Clearly, further studies on different optic nerve diseases using large sample sizes are necessary. Presently, we conclude that the PERG, while an interesting measurement, has limited value in the detection of optic nerve demyelination and does not compare with the PVEP.

Table 2: Mean P100 peak latency over $\mathrm{O}_{1}$ and $\mathrm{O}_{2}$ as a function of check size in normal control and MS groups after monocular stimulation of left and right eyes.

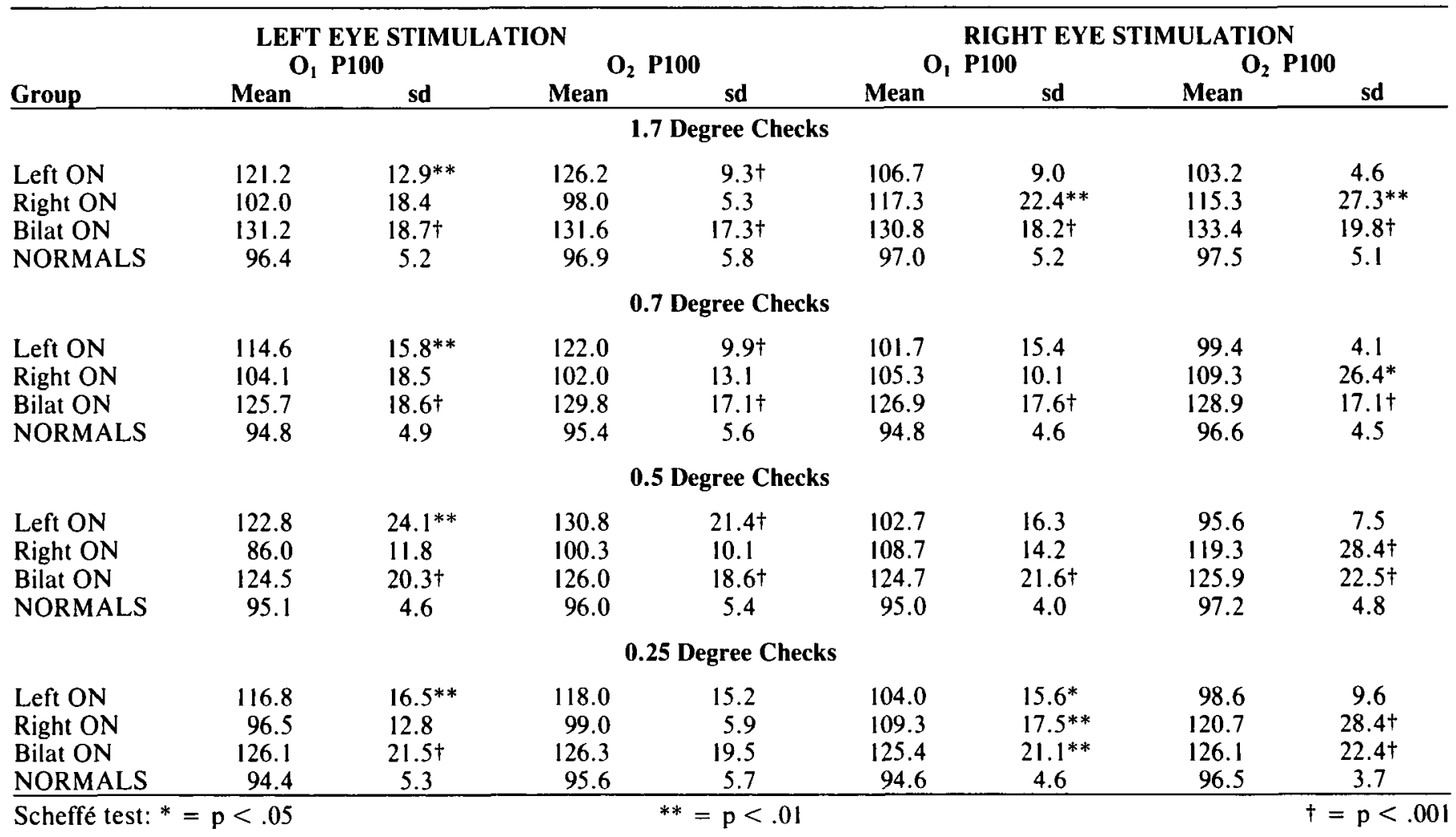

Table 3: The relative frequency of PERG and PVEP delay in a sample population of MS patients $(\mathbf{N}=\mathbf{2 8})$.

\begin{tabular}{cccc}
\hline $\begin{array}{c}\text { Check size } \\
\text { (deg/side) }\end{array}$ & $\begin{array}{c}\text { Normal } \\
\text { PERG } \\
\text { and PVEP }\end{array}$ & $\begin{array}{c}\text { PVEP } \\
\text { delayed }\end{array}$ & $\begin{array}{c}\text { Proportion of VEP } \\
\text { delay cases with } \\
\text { PERG delay }\end{array}$ \\
\hline 1.7 & $0(0 \%)$ & $27 / 28(96 \%)$ & $8 / 27(30 \%)$ \\
.7 & $1 / 28(4 \%)$ & $26 / 28(93 \%)$ & $6 / 26(23 \%)$ \\
.5 & $2 / 28(7 \%)$ & $25 / 28(90 \%)$ & $10 / 25(40 \%)$ \\
.25 & $3 / 28(11 \%)$ & $24 / 28(86 \%)$ & $10 / 24(42 \%)$ \\
\hline
\end{tabular}




\section{ACKNOWLEDGEMENTS}

Aided by a grant from the Canadian National Institute for the Blind out of the E. A. Baker Foundation. The Town of Mount Royal Old Timers Executive Hockey League, St. Nicholas and St. Georges Men's Club and Kiwanis Club of Royal Mount gave generous assistance for the purchase of the equipment. Judy Bengle, R.N. gave invaluable technical assistance.

\section{REFERENCES}

Arden, G.B., Vaegan and Hogg, C.R. (1982). Clinical and experimental evidence that the pattern electroretinogram (PERG) is generated in more proximal retinal layers than the focal electroretinogram (FERG). Ann. New York Acad. Sci. 388:580-601.

Bobak, P., Bodis-Wollner, I. and Harnois, C. (1983). Simultaneously recorded pattern-reversal electroretinograms and visual evoked potentials in the evaluation of different types of optic nerve damage. Ann. Neurol. 14:147.

Coupland, S.G. and Kirkham. T.H. (1981). Electroretinograhy and nystagmus: subtraction of eye movement artefact. Canad. J. Ophthalmol. 16:192-194.

Dawson, W., Maida, T.M. and Rubin, M.L. (1982). Human patternevoked retinal responses are altered by optic atrophy. Invest. Ophthalmol. Vis. Sci. 22:796-803.
Fiorentini, A., Maffei, L., Pirchio, M., Spinelli, D. and Porciatti, V. (1981). The ERG in response to alternating gratings in patients with diseases of the peripheral visual pathway. Invest. Ophthalmol. Vis. Sci. 21:490-493.

Kirkham, T.H. and Coupland, S.G. (1981a). Multiple regression analysis of diagnostic predictors in optic nerve disease. Canad. J. Neurol. Sci. 8:67-72.

Kirkham, T.H. and Coupland, S.G. (1981b). Abnormal pattern electroretinograms with macular cherry-red spots: evidence for selective ganglion cell damage. Current Eye Res. 1:367-372.

Kirkham, T.H. and Coupland, S.G. (1983). Pattern ERG's and check size: absence of spatial frequency tuning. Current Eye Res. 2: $511-521$.

Maffei, L. (1982). Electroretinographic and visual cortical potentials in response to alternate gratings. Ann. New York Acad. Sci. 388:1-10.

Maffei, L. and Fiorentini, A. (1981). Electroretinographic responses to alternate gratings before and after section of the optic nerve. Science 211:953-955.

May, J.G., Ralston, J.V., Reed, J.L. and Van Dyk, H.J.L. (1982). Loss in pattern-elicited electroretinograms in optic nerve dysfunction. Am. J. Ophthalmol. 93:418-422.

McDonald, W.I. and Halliday, A.M. (1977). Diagnosis and classification of multiple sclerosis. Brit. Med. Bull. 33:4-8.

Sherman, J. (1982). Simultaneous pattern-reversal electroretinograms and visual evoked potentials in disease of the macula and optic nerve. Ann. New York Acad. Sci. 388:214-226. 